\title{
Surface Plasmon Resonance Sensitivity of Metal Nanostructures: Physical Basis and Universal Scaling in Metal Nanoshells
}

\author{
Prashant K. Jain ${ }^{\dagger}$ and Mostafa A. El-Sayed ${ }^{*}, \dagger$ \\ Laser Dynamics Laboratory, School of Chemistry and Biochemistry, Georgia Institute of \\ Technology, Atlanta, Georgia 30332-0400. \\ SUPPORTING INFORMATION
}

* Corresponding author. E-mail: melsayed@gatech.edu. Tel: 404-894-0292. Fax: 404-894-0294.

† Georgia Institute of Technology. 


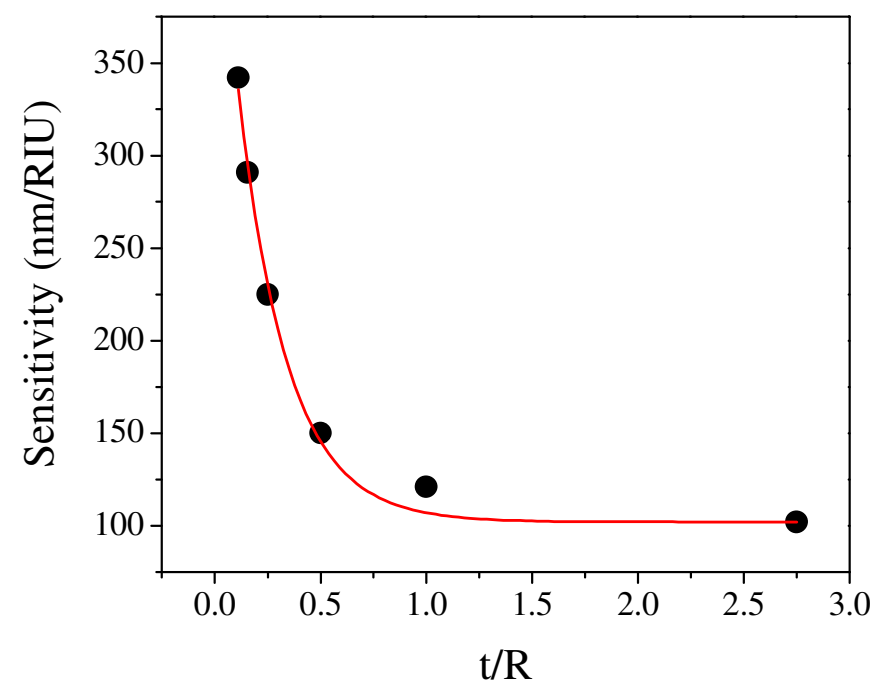

Fig. S1. Sensitivity $\left(\Delta \lambda_{s p} / \Delta n_{m}\right)$ plotted as a function of the shell thickness-to-core radius ratio $(t / R)$ for $60-\mathrm{nm}$ silica core-gold nanoshell. The sensitivity falls near-exponentially with increasing $t / R$ as seen from the fit (solid line) to the single-exponential decay $y=y_{0}$ $+a . \exp (-x / \tau)$ with $\tau=0.23 \pm 0.02, a=378 \pm 25$ and $y_{0}=102 \mathrm{~nm} / \mathrm{RIU}$. 


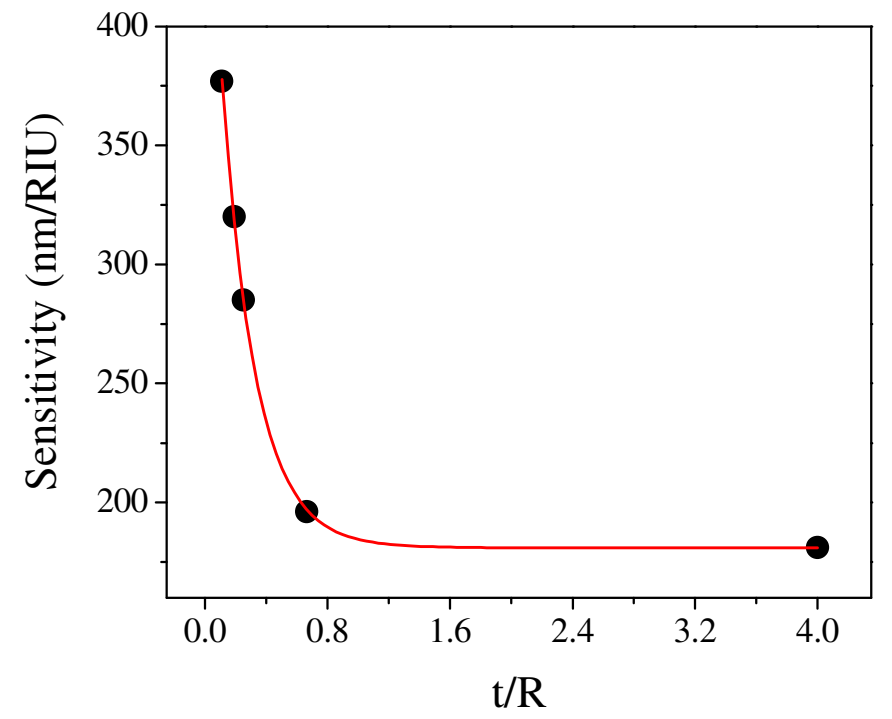

Fig. S2. Sensitivity $\left(\Delta \lambda_{s p} / \Delta n_{m}\right)$ plotted as a function of the shell thickness-to-core radius ratio $(t / R)$ for $100-n m$ silica core-gold nanoshell. The sensitivity falls near-exponentially with increasing $t / R$ as seen from the fit (solid line) to the single-exponential decay $y=y_{0}$ $+a \cdot \exp (-x / \tau)$ with $\tau=0.22 \pm 0.00, a=326 \pm 4$ and $y_{0}=181 \mathrm{~nm} / \mathrm{RIU}$. 


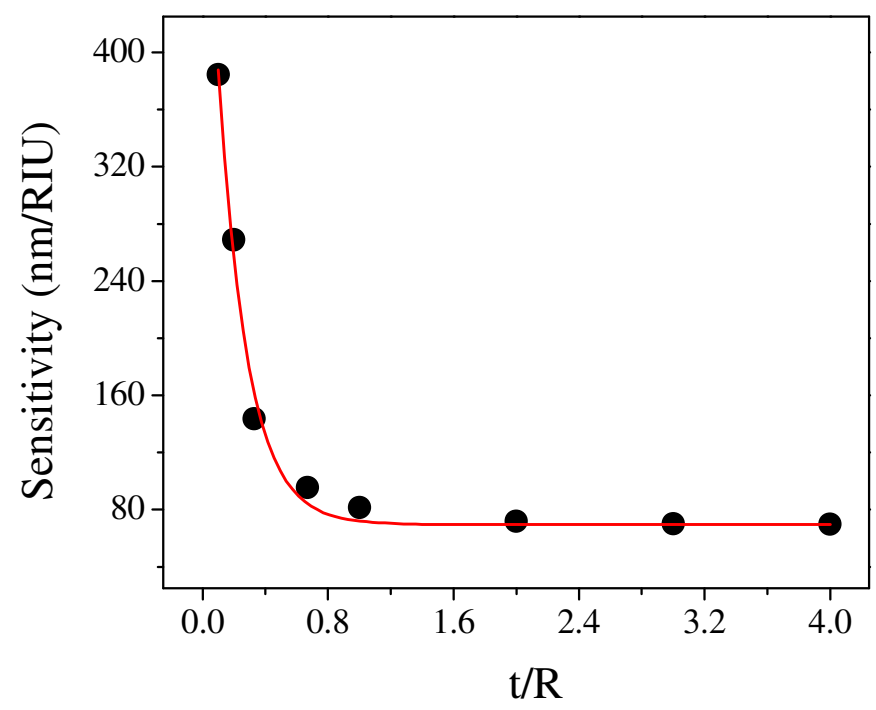

Fig. S3. Sensitivity $\left(\Delta \lambda_{s p} / \Delta n_{m}\right)$ plotted as a function of the shell thickness-to-core radius ratio $(t / R)$ calculated from dipolar quasistatic approximation for a silica core-gold nanoshell. The sensitivity falls near-exponentially with increasing $t / R$ as seen from the fit (solid line) to the single-exponential decay $y=y_{0}+a \cdot \exp (-x / \tau)$ with $\tau=0.18 \pm 0.01, a=$ $547 \pm 37$ and $y_{0}=70 \mathrm{~nm} / \mathrm{RIU}$. 


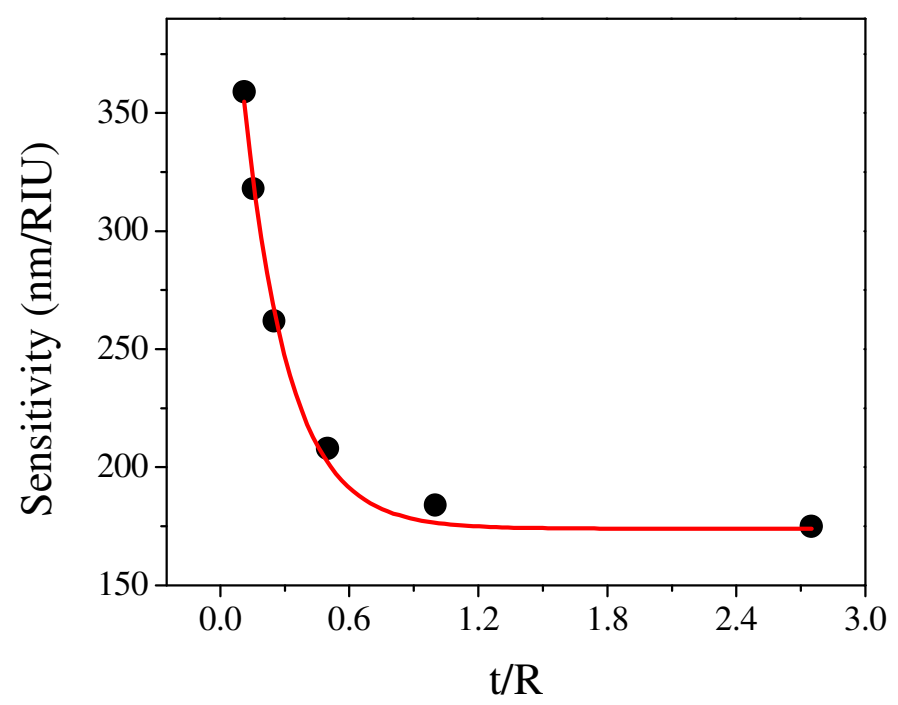

Fig. S4. Sensitivity $\left(\Delta \lambda_{s p} / \Delta n_{m}\right)$ plotted as a function of the shell thickness-to-core radius ratio $(t / R)$ for 60 -nm silica-core silver nanoshell. The sensitivity falls near-exponentially with increasing $t / R$ as seen from the fit (solid line) to the single-exponential decay $y=y_{0}$ $+a \cdot \exp (-x / \tau)$ with $\tau=0.21 \pm 0.02, a=308 \pm 19$ and $y_{0}=174 \mathrm{~nm} / \mathrm{RIU}$. 


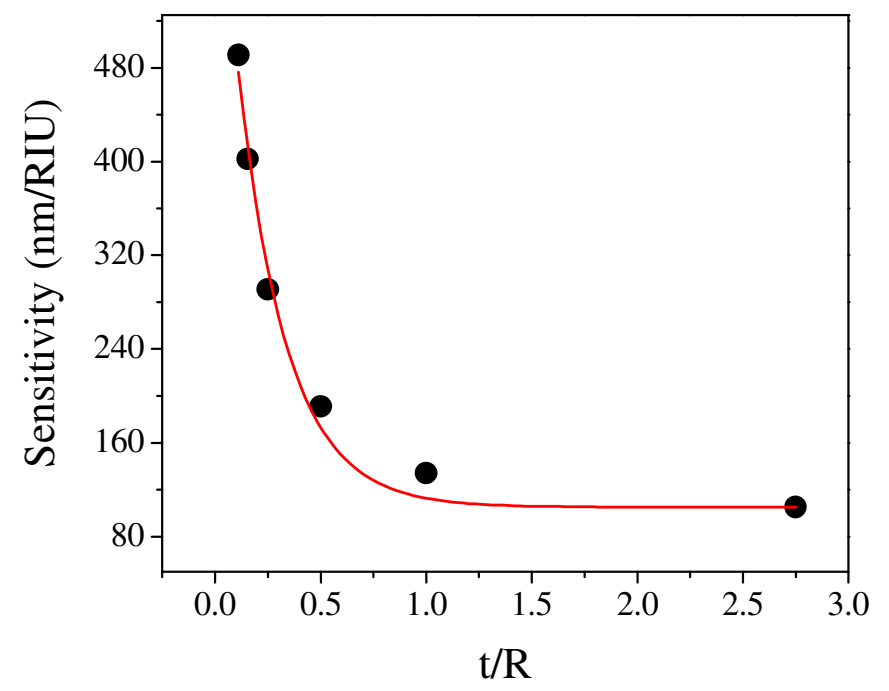

Fig. S5. Sensitivity $\left(\Delta \lambda_{s p} / \Delta n_{m}\right)$ plotted as a function of the shell thickness-to-core radius ratio $(t / R)$ for 60 -nm hollow core-gold nanoshell. The sensitivity falls near-exponentially with increasing $t / R$ as seen from the fit (solid line) to the single-exponential decay $y=y_{0}$ $+a \cdot \exp (-x / \tau)$ with $\tau=0.23 \pm 0.03, a=603 \pm 53$ and $y_{0}=105 \mathrm{~nm} / \mathrm{RIU}$. 